\title{
CAMBIOS ENTESIALES EN UNA MUESTRA BIOARQUEOLÓGICA DEL NORESTE DE PATAGONIA: PRIMEROS RESULTADOS DE UNA COMPARACIÓN METODOLÓGICA
}

\author{
ENTHESEAL CHANGES IN A BIOARCHAEOLOGICAL SAMPLE FROM THE \\ NORTHEAST OF PATAGONIA: FIRST RESULTS OF A METHODOLOGICAL \\ COMPARISON
}

Victoria Romano ${ }^{*}$ y Alejandro Serna ${ }^{1,2}$

${ }^{1}$ División Arqueología. Facultad de Ciencias Naturales y Museo. Universidad Nacional de La Plata. La Plata. Argentina

${ }^{2}$ Consejo Nacional de Investigaciones Científicas y Tecnológicas (CONICET). La Plata. Argentina

PALABRAS CLAVE: cambios entesiales; marcadores de actividad; método de Hawkey y Merbs; método Coimbra RESUMEN En este trabajo se presenta la primera aproxi-
mación al estudio de cambios entesiales sobre restos hu-
manos procedentes del noreste de Patagonia. Teniendo en
cuenta que dos de los métodos de registro frecuentemente
utilizados están basados en supuestos diferentes, "Hawkey
y Merbs" y "Coimbra", se propone comparar y discutir los
resultados obtenidos a partir de la aplicación de ambos y
la implementación de una nueva medida de cuantificación.
Para ello, se analizan elementos óseos del miembro superior e inferior en una muestra procedente del valle medio del río Negro. Se calculó el error intraobservador y el análisis de los datos se basó en dos medidas de cuantificación: la presencia de al menos un cambio y el Índice de Afectación. La comparación entre métodos muestra tendencias diferentes. Mediante Hawkey y Merbs no se observa una tendencia clara de afectación, y desde Coimbra un mayor compromiso del miembro inferior. Rev Arg Antrop Biol 22(1), 2020. doi: $10.24215 / 18536387 \mathrm{e} 012$

KEYWORDS entheseal changes; activity markers; Hawkey and Merbs method; Coimbra method

ABSTRACT This work presents the first approach to the study of entheseal changes in human remains from northeastern Patagonia. Taking into account that two of the most widely used registration methods are based on different assumptions, "Hawkey and Merbs" and "Coimbra", it is proposed to compare and discuss the results obtained by the application of both and the application of a new measure of quantification. With this purpose, a sample composed by upper and lower human limb bones from the middle Negro river valley were analyzed. The intraobserver error was estimated, and the data analysis was based on two quantification measures: the presence of at least one change, and the affectation index. The comparison between methods shows different trends. Although Hawkey and Merbs does not show tendency of affectation, the Coimbra method is consistent and indicates a greater commitment of the lower extremity. Rev Arg Antrop Biol 22(1), 2020. doi: $10.24215 / 18536387 \mathrm{e} 012$
Las entesis son áreas donde los tendones, ligamentos o músculos se insertan sobre el hueso. En estas áreas es posible caracterizar y cuantificar alteraciones morfológicas o "cambios entesiales" (CE), que son utilizados en bioarqueología con el objetivo de estudiar la actividad física y las demandas mecánicas del cuerpo (Benjamin et al., 2002; Henderson, Mariotti, Santos, Villotte y Wilczak, 2017; Villote et al., 2016). Si bien se sabe que los CE se relacionan con la actividad física, se ha reconocido que tienen una etiología multifactorial dependiendo también de la edad, el metabolismo, la masa corporal, la constitución genética y distintos tipos de patologías (Henderson et al., 2017; Villotte y Knüsel, 2013). La naturaleza de las entesis es otro factor importante, ya que de su composición histológica depende la expresión y el motivo por el que se producen los cambios. En las entesis fibrosas el tendón o músculo se inserta directamente al periostio o al hueso, mientras que las fibrocartilaginosas son más complejas e involucran distintos tejidos (Benjamin et al., 2002; Villotte y Knüsel, 2013). Las primeras, se localizan generalmente en las diáfisis y debido

\footnotetext{
Financiamiento: Agencia Nacional de Promoción Científica y Tecnológica. PICT 2015-3645.

*Correspondencia a: Victoria Romano. Unidad de Investigación Anexo Museo. Laboratorio N²128. Calle 60 y 122. 1900 La Plata. Argentina.

E-mail: romanovictoriarv@gmail.com
}

Recibido 13 Febrero 2019; aceptado 27 Junio 2019

doi: $10.24215 / 18536387 \mathrm{e} 012$ 
a que se conoce poco sobre su anatomía se cree que sus alteraciones se relacionan principalmente con la edad y no está claro el potencial impacto de la actividad física sobre ellas. En cambio, las entesis fibrocartilaginosas suelen encontrarse en las epífisis -porciones sometidas a mayor estrésy se conoce con mayor profundidad su anatomía, por lo que es posible relacionar sus cambios morfológicos a las exigencias de la actividad física (Benjamin et al., 2002; Benjamin y McGonagle, 2001, 2009; Villotte y Knüsel, 2013).

Para relevar los CE en los restos óseos humanos se han elaborado distintas metodologías (e.g., Hawkey y Merbs, 1995; Henderson, Mariotti, Pany-Kucera, Villotte y Wilczak, 2013, 2016; Mariotti, Facchini y Belcastro, 2007; Robb, 1998; Villote et al., 2010). Este trabajo se enfoca en dos métodos de amplia utilización en la investigación bioarqueológica, el método "Hawkey y Merbs" (HyM) (Hawkey y Merbs, 1995) y el "método Coimbra" (Co) (Henderson et al., 2013, 2016). El primero, elaborado por Hawkey (1988) y posteriormente publicado por Hawkey y Merbs (1995), estudia entesis fibrosas y fibrocartilaginosas mediante el registro de cambios proliferativos y erosivos a los que relaciona con un tipo y grado de estrés mecánico. El segundo método, publicado por el grupo de la Universidad de Coimbra (Coimbra, Portugal) en el año 2013 (Henderson et al., 2013) y posteriormente con modificaciones en el año 2016 (Henderson et al., 2016), fue elaborado sobre una muestra con sexo, edad y ocupación conocidas, y propone estudiar únicamente los CE sobre las fibrocartilaginosas. Esta metodología selecciona tipos distintos de cambios proliferativos, erosivos y texturales, a los que por sí mismos no asigna una interpretación específica, en términos de demanda mecánica (Henderson et al., 2016). En este trabajo de carácter exploratorio se propone, por un lado, observar las similitudes y diferencias de los resultados de los métodos HyM y Co y; por el otro, utilizar una nueva medida de análisis (Índice de Afectación), con el fin de planificar futuros estudios macroescalares con muestras mayores. Para ello, se analizarán elementos del miembro superior e inferior bajo distintos niveles de detalle anatómico (i.e., entesis en particular, complejo funcional y miembro) en una muestra de restos humanos procedentes del sitio arqueológico Negro Muerto 3, ubicado en el valle medio del río Negro (provincia de Río Negro) (Fig. 1).

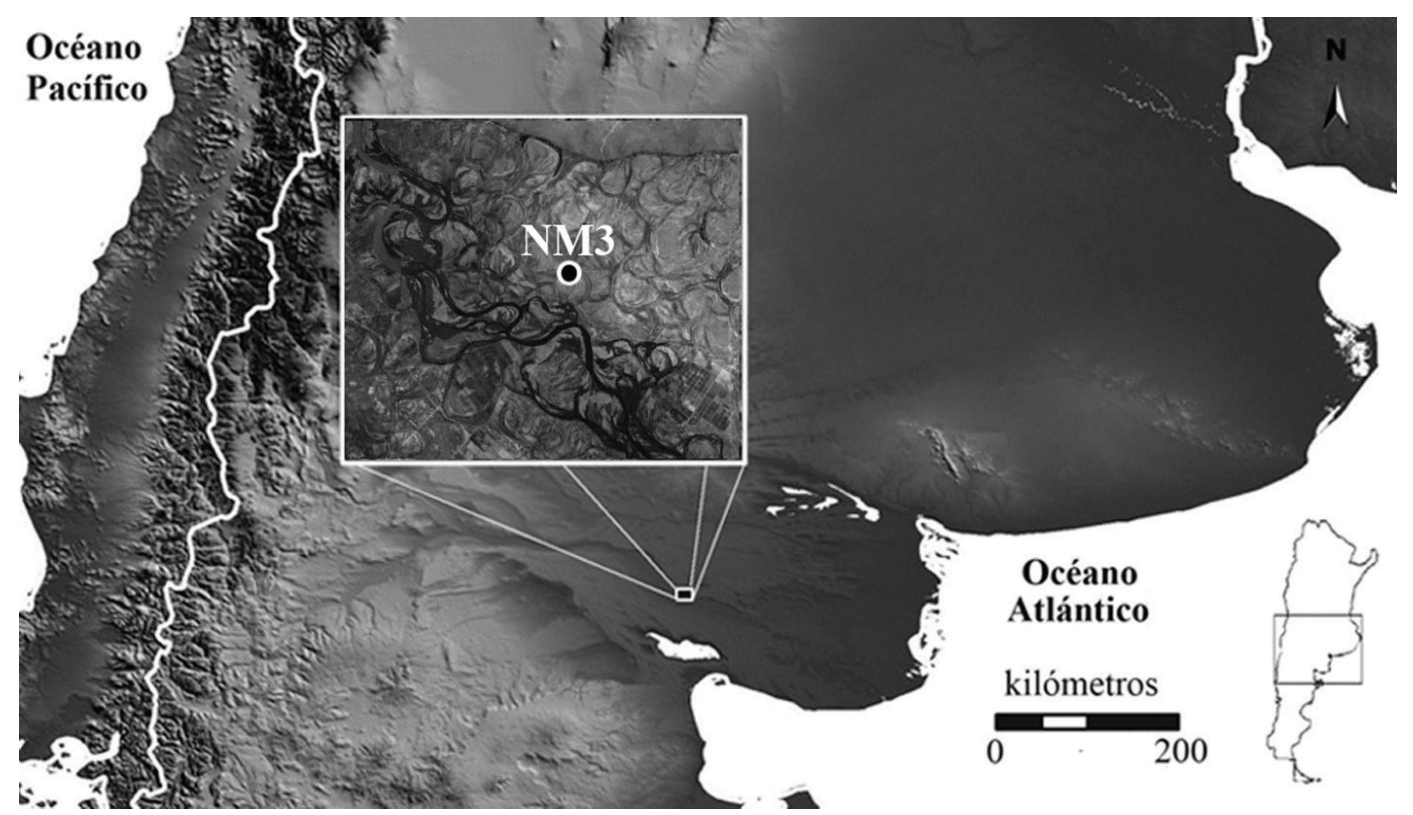

Fig. 1. Ubicación del sitio arqueológico Negro Muerto 3 (NM3). 


\section{MATERIAL Y MÉTODOS}

\section{Muestra}

La muestra aquí analizada proviene del sitio arqueológico Negro Muerto 3 (NM3), localizado en el valle medio del río Negro (provincia de Río Negro) (Fig. 1) y fechado para el Holoceno tardío. En el mismo se hallaron artefactos líticos, material faunístico, alfarería y restos humanos recuperados in situ y en superficie (Prates et al., 2019; Serna y Romano, 2018). En este trabajo se estudiaron ocho individuos adultos (tres de edad específica indeterminada y cinco de entre 20-39 años) de ambos sexos (seis masculinos y dos femeninos) (véase detalle de la representación anatómica en Material Suplementario 1 y 2). La determinación sexo-etaria se llevó a cabo siguiendo metodologías bioarqueológicas estándares (Buikstra y Ubelaker, 1994; Scheuer y Black, 2000; White y Folkens, 2005), basadas en el estudio morfoscópico y morfométrico de los elementos óseos que mejor expresan el dimorfismo sexual y aquellos que permiten precisar la edad mediante grados de fusión ósea, erupción dentaria, características morfológicas generales y métricas, cuyos resultados ya han sido publicados (Prates et al., 2019; Serna y Romano, 2018).

\section{Metodología}

El registro de $\mathrm{CE}$ se realizó siguiendo dos propuestas. Por un lado, el método de Hawkey y Merbs (1995) (HyM), que estudia ambos tipos de entesis sobre las que analiza tres cambios (robusticidad -ROB-, osificación -OS- y lesión de estrés -LE-) y puntúa de acuerdo con una escala de severidad que refiere al impacto del estrés mecánico $(0=$ ausente, $1=$ leve, $2=$ moderado y 3 =severo). Por el otro lado, se aplicó el método Coimbra (Co) (Henderson et al., 2016), que estudia únicamente entesis fibrocartilaginosas y divide cada una de ellas en una "zona 1" (margen opuesto a la dirección en que se insertan las fibras del músculo) y una "zona 2" (margen y superficie entesial restante). En las zonas se relevan distintos cambios, en la zona 1 se considera la formación ósea (FO1) y la erosión (ER1) y, en la zona 2, la formación ósea
(FO2), erosión (ER2), cambio de textura (CT), porosidad fina (POF), macroporosidad (MPO) y cavitación (CA). Todos estos rasgos son definidos de acuerdo a una escala de expresión (0-2), a excepción de CT que es relevado de según presencia/ausencia (Henderson et al., 2016).

\section{Procesamiento y análisis de datos}

El relevamiento de los CE se realizó por el mismo observador (VR) en dos ocasiones con cada método y se testeó el error intraobservador mediante el índice Kappa $(k)$, que proporciona el grado de acuerdo entre pares de observaciones por encima del esperado por azar (Bernal, González, Pérez y Del Papa, 2004; Cohen, 1960). Para el relevamiento de los CE se consideraron entesis de los miembros superiores e inferiores, 17 en el método HyM y 16 para el método Co (Tabla 1). El análisis de los datos tuvo en cuenta distintos niveles anatómicos: entesis individual, complejo funcional -conjunto de entesis que pertenecen a músculos o ligamentos que participan en la funcionalidad de un complejo articular particular-, y entesis agrupadas a nivel de miembros, considerando que los músculos actúan en grupos y no individualmente (Kapandji, 2006; Stirland, 1998; Testut y Latarjet, 1954)

El procesamiento y análisis de datos involucró dos medidas diferentes. Por un lado, se contabilizó la frecuencia de cada CE y la frecuencia de entesis con al menos un cambio $(\mathrm{n} / \mathrm{N})$. Por otro lado, se calculó el Índice de Afectación (IA), definido como el cociente entre la cantidad de cambios presentes en una entesis/conjunto de entesis y el máximo de cambios que dicha/ conjunto podría presentar de acuerdo al método utilizado. Por ejemplo: si se analizan desde Co (que registra un máximo de ocho cambios), 12 entesis del músculo Subescapular, la cantidad máxima de $\mathrm{CE}$ que puede presentar ese grupo es de $96(12 * 8=96)$, siendo ese el grado de afección máxima. Si en dicho conjunto se observaran 20 cambios, el IA sería de 0,208 o $21 \%$. Por último, se analizó la presencia de cambios $(\mathrm{n} / \mathrm{N})$ por lateralidad a nivel de entesis, complejo funcional y miembro mediante el cálculo de $\chi^{2}$ (Daniel, 1987). El nivel de significación de todos los estadísticos fue $\alpha=0,05$. Los análisis fueron realizados con SPSS 25 y PAST 3.22. 
TABLA 1. Entesis consideradas desde cada método en relación al miembro y complejo funcional en el que participan y elemento óseo en que se encuentran

\begin{tabular}{|c|c|c|c|}
\hline Miembro & $\begin{array}{l}\text { Complejo } \\
\text { funcional }\end{array}$ & Método Hawkey y Merbs & Método Coimbra \\
\hline \multirow{9}{*}{ Superior } & \multirow{4}{*}{ Hombro } & Deltoides (Húmero) & Subescapular (Húmero) \\
\hline & & Dorsal ancho (Húmero) & Supraespinoso-infraespinoso (Húmero) \\
\hline & & Pectoral mayor (Húmero) & Redondo menor (Húmero) \\
\hline & & Redondo mayor (Húmero) & \\
\hline & \multirow{3}{*}{ Codo } & Braquiorradial (Húmero) & Tríceps Braquial (Cúbito) \\
\hline & & Braquial anterior (Cúbito) & Braquial anterior (Cúbito) \\
\hline & & Bíceps braquial (Radio) & Bíceps braquial (Radio) \\
\hline & \multirow{2}{*}{ Muñeca-mano } & Pronador cuadrado (Cúbito) & Tendón común extensores (Húmero) \\
\hline & & Supinador (Cúbito) & Tendón común flexores (Húmero) \\
\hline \multirow{11}{*}{ Inferior } & \multirow{4}{*}{ Cadera } & \multirow{2}{*}{ Glúteo mayor (Fémur) } & Iliopsoas (Fémur) \\
\hline & & & Glúteo menor (Fémur) \\
\hline & & \multirow{2}{*}{ Tuberosidad isquiática (Isquion)* } & Glúteo medio (Fémur) \\
\hline & & & Semimembranoso-semitendinoso (Isquion) \\
\hline & \multirow{5}{*}{ Rodilla } & Tuberosidad isquiática (Isquion) & Semimembranoso-semitendinoso (Isquion) \\
\hline & & Línea áspera $\left(\right.$ Fémur) ${ }^{* *}$ & 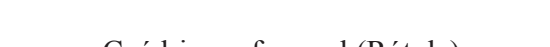 \\
\hline & & Gastrocnemios (Fémur) & Cuadriceps temoral (Rotula) \\
\hline & & Poplíteo (Tibia) & Poplíteo (Fémur) \\
\hline & & Ligamento rotuliano (Tibia) & Ligamento rotuliano (Tibia) \\
\hline & \multirow{2}{*}{ Tobillo-pie } & Soleo (Tibia) & Tríceps sural (Calcáneo) \\
\hline & & Aponeurosis plantar (Calcáneo) & \\
\hline
\end{tabular}

${ }^{*}$ Tuberosidad isquiática: origen del bíceps femoral, semimembranoso y semitendinoso; **Línea áspera: origen de bíceps femoral (porción corta), vasto medial y vasto lateral e inserción del aductor menor, aductor mayor y aductor largo.

\section{RESULTADOS}

\section{Error intraobservador}

Los índices de Kappa $(k)$ calculados indican, para HyM $k=0,93$ y para Co $k=0,88$. En base a la escala interpretativa propuesta por Landis y Koch (1977), ambos valores muestran muy buena concordancia, por lo que el error intraobservador es poco significativo.

\section{Método Hawkey y Merbs}

Mediante el método HyM se analizó un total de 201 entesis, de las cuales el 52\% presenta al menos un cambio $(\mathrm{n} / \mathrm{N})$. La cantidad de cambios registrada fue de 140 (IA=23\%); un $83 \%$ en es- tado leve, un $17 \%$ moderado y no hay ningún caso severo. El cambio entesial más frecuente es la ROB con una frecuencia de $40 \%(\mathrm{n} / \mathrm{N})$ (Tabla 2). Las entesis con mayor presencia de cambios son del glúteo mayor (Fig. 2a) y del braquiorradial ( $\mathrm{n} / \mathrm{N} \geq 83 \%$ ), y con mayor afectación por cantidad de $\mathrm{CE}$ las del glúteo mayor (IA=48\%), también se destacan las entesis del pectoral mayor y de la tuberosidad isquiática (IA=31\%). La entesis menos afectada tanto por presencia de CE como por el IA son del ligamento rotuliano $(\mathrm{n} / \mathrm{N}=30 \%$; IA=10\%) (Fig. 3a). El complejo funcional más afectado es el de cadera, tanto por presencia como por el Índice de Afectación (n/ $\mathrm{N}=71 \%$; IA=39\%). Los complejos del miembro superior (hombro, codo y muñeca) muestran un porcentaje de presencia de cambios $(\mathrm{n} / \mathrm{N})$ 
TABLA 2. Frecuencia de cambios entesiales relevados desde el método de Hawkey y Merbs (1995)

\begin{tabular}{ccccc}
\hline Entesis & N & Cambios entesiales & \\
& & Robusticidad & Lesión de estrés & Osificación \\
\hline Deltoides & 12 & $6(50 \%)$ & $1(8 \%)$ & $2(17 \%)$ \\
Dorsal ancho & 12 & $4(33 \%)$ & $2(17 \%)$ & 0 \\
Pectoral mayor & 12 & $8(67 \%)$ & $1(8 \%)$ & $2(17 \%)$ \\
Redondo mayor & 11 & $4(37 \%)$ & $1(9 \%)$ & 0 \\
Braquiorradial & 12 & $2(17 \%)$ & 0 & $8(67 \%)$ \\
Braquial anterior & 13 & $5(39 \%)$ & 0 & $6(46 \%)$ \\
Bíceps braquial & 12 & $5(42 \%)$ & 0 & $1(8 \%)$ \\
Pronador cuadrado & 12 & $4(33 \%)$ & 0 & $2(16 \%)$ \\
Supinador & 10 & $2(20 \%)$ & 0 & $6(60 \%)$ \\
Glúteo mayor & 14 & $12(86 \%)$ & 0 & $8(57 \%)$ \\
Tuberosidad isquiática & 14 & $8(57 \%)$ & 0 & $5(36 \%)$ \\
Línea áspera & 14 & $5(36 \%)$ & 0 & $4(29 \%)$ \\
Gastrocnemios & 12 & $4(33 \%)$ & 0 & 0 \\
Ligamento rotuliano & 10 & $3(30 \%)$ & 0 & $3(27 \%)$ \\
Poplíteo & 11 & $4(36 \%)$ & 0 & $2(18 \%)$ \\
Soleo & 11 & $5(45 \%)$ & $1(9 \%)$ & 0 \\
Aponeurosis plantar & 9 & $1(11 \%)$ & $6(3 \%)$ & 0 \\
\hline Total & 201 & $\mathbf{8 2}(\mathbf{4 0 \% )}$ & & $0 \%)$ \\
\hline
\end{tabular}

En negrita el resultado de mayor representación.

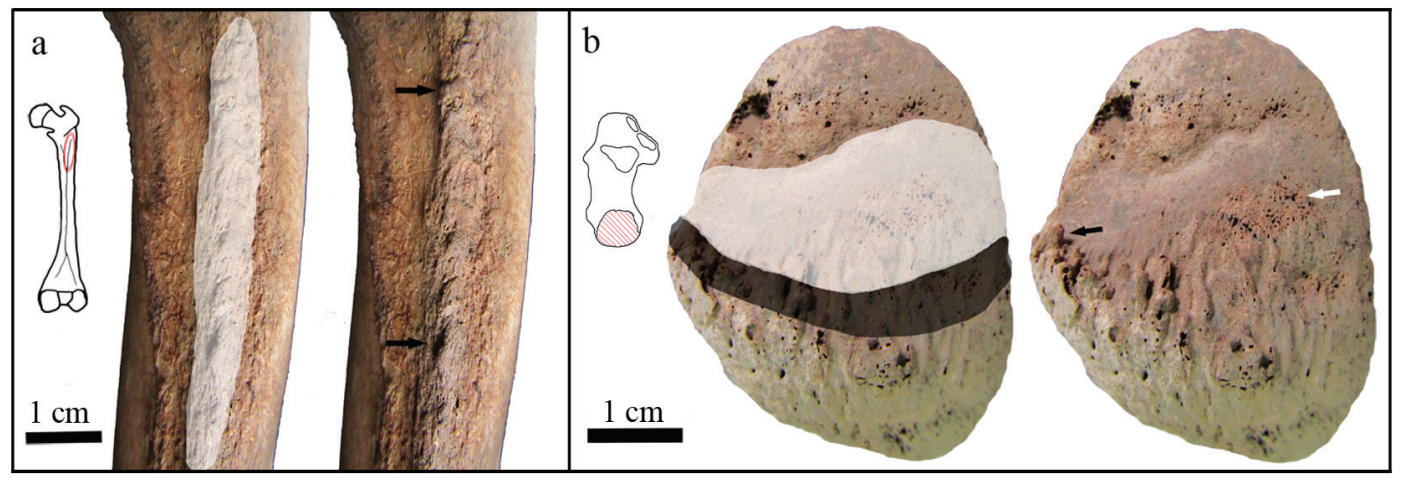

Fig. 2. Demarcación de las entesis y señalización de cambios entesiales. a) Entesis fibrosa del glúteo mayor derecho analizada desde Hawkey y Merbs. IZQ: demarcación del área entesial; DER: cambios entesiales: robusticidad moderada y osificación leve (flechas negras). b) Entesis fibrocartilaginosa del tríceps sural izquierdo analizada desde Coimbra. IZQ: demarcación del área entesial, zona 1 (en negro) y zona 2 (en blanco); DER: cambios entesiales: formación ósea en zona 1 con grado 2 (flecha negra) y porosidad fina grado 1 (flecha blanca). 
entre 50-60\%, algo mayor al valor de la rodilla y tobillo-pie que rondan entre $40-45 \% \mathrm{y}$, en cuanto al IA, todos los complejos (a excepción de la cadera) coincidieron en 21,5\% ( $\pm 1 \%)$ (Fig. $3 b)$. Agrupando las entesis a nivel de miembros superiores e inferiores se observan resultados semejantes tanto para la presencia de cambios (ca. 50\%), como para el IA (ca. 24\%) (Fig. 3c). Las entesis más afectadas por complejo funcional son las del pectoral mayor (hombro), braquiorradial (codo), supinador (muñeca-mano), glúteo mayor (cadera), tuberosidad isquiática (rodilla) y sóleo (tobillo-pie). Con respecto a la presencia de cambios por lateralidad no se observó diferencias significativas a nivel de entesis, complejos funcionales, ni de miembros (Tabla 3).

\section{Método Coimbra}

Se analizó un total de 177 entesis, de las cuales el $69 \%$ presenta al menos un cambio $(\mathrm{n} / \mathrm{N})$, la cantidad de cambios registrada fue de 213 (IA=15\%). El 81\% de los cambios registrados conserva la expresión en grado 1 y el $19 \%$ en grado 2 (sin considerar el CT que solo se releva su presencia). El cambio entesial más frecuente es la ER2 (31\%) (Tabla 4). Las entesis con mayor presencia de cambios son las del tríceps sural (Fig. 2b), cuádriceps femoral, glúteo menor y poplíteo ( $\mathrm{n} / \mathrm{N} \geq 91 \%$ ), aunque también se destacan las del bíceps braquial, semimembranososemitendinoso e iliopsoas ( $\mathrm{n} / \mathrm{N} \geq 83 \%$ ). Las entesis con mayor IA son las del semimembranososemitendinoso ( $\mathrm{IA}=30 \%$ ), tríceps sural y glúteo menor $(\mathrm{IA}=25 \%)$. Las entesis menos afectadas son del tendón común de los flexores ( $\mathrm{n} / \mathrm{N}=31 \%$; $\mathrm{IA}=5 \%)$ y la del glúteo medio $(\mathrm{n} / \mathrm{N}=31 \%$; $\mathrm{IA}=7 \%$ ) (Fig. 4a). En relación a los complejos funcionales, el más afectado por ambos valores es el tobillo-pie ( $\mathrm{n} / \mathrm{N}=100 \% ; \mathrm{IA}=25 \%$ ), seguido por la rodilla ( $\mathrm{n} / \mathrm{N}=84 \%$; IA $=21 \%$ ) y el complejo menos afectado es la muñeca-mano (n/N=39\%; $\mathrm{IA}=6 \%$ ) (Fig. $4 \mathrm{~b}$ ). El complejo del hombro y el codo tienen resultados muy semejantes $(\mathrm{n} / \mathrm{N}=67$ $\pm 1 \%$; IA $=13 \pm 1 \%$ ), y el de la cadera se encuentra en un punto intermedio entre todos los complejos ( $\mathrm{n} / \mathrm{N}=73 \%$; IA=19\%). A nivel de miembros se observa que el inferior está más afectado $(\mathrm{n} /$ $\mathrm{N}=80 \% ; \mathrm{IA}=21 \%$ ) que el superior $(\mathrm{n} / \mathrm{N}=60 \%$; $\mathrm{IA}=11 \%$ ) (Fig. 4c). Las entesis más afectadas por complejo funcional son la del subescapular (hombro), tríceps braquial (codo), tendón común de los extensores (muñeca-mano), glúteo me-

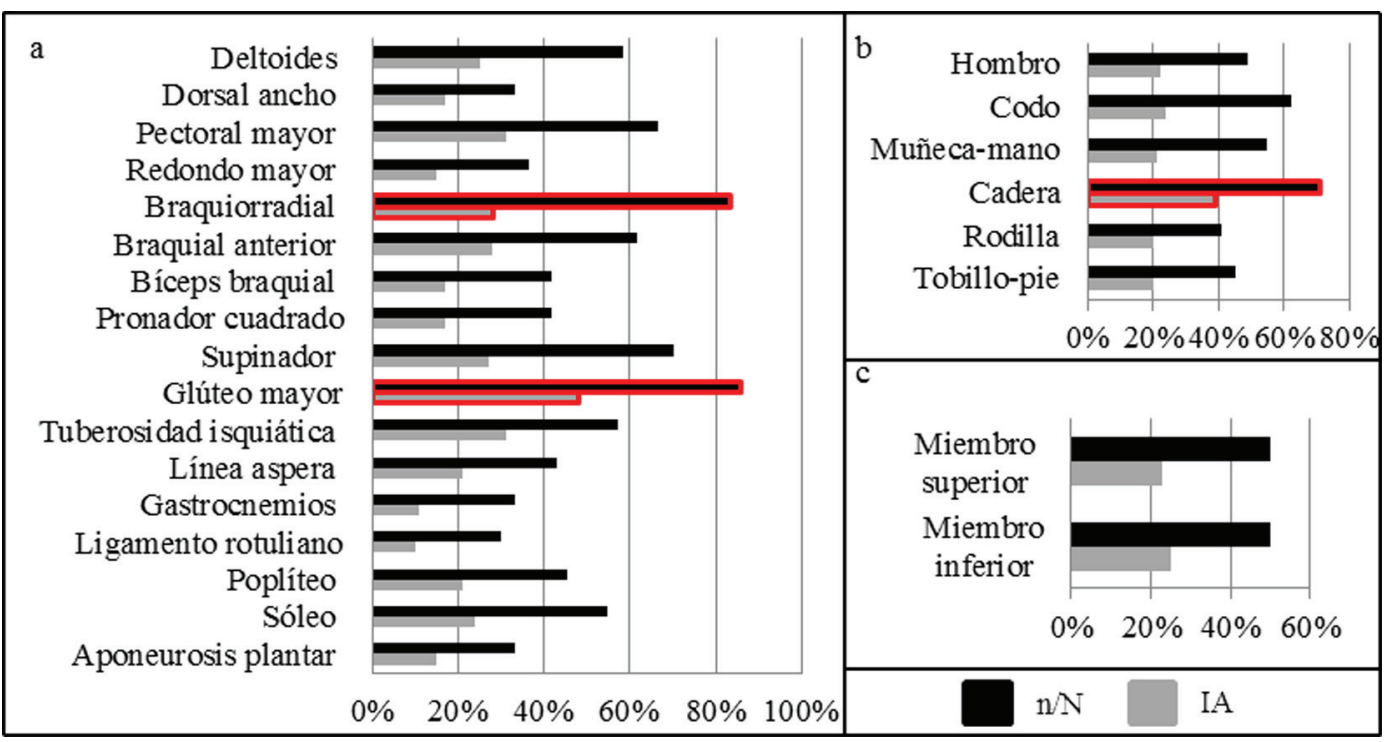

Fig. 3. Resultados desde el método Hawkey y Merbs (1995) de presencia de cambios (n/N) e Índice de Afectación (IA) a distintos niveles: a) entesis; b) complejo funcional; c) miembros. En color algunos porcentajes elevados que serán discutidos especialmente. 
TABLA 3. Valores de $\chi^{2}$ para la presencia de cambios por lateralidad en el método de Hawkey y Merbs (1995)

\begin{tabular}{|c|c|c|c|c|c|}
\hline & \multicolumn{5}{|c|}{$\mathrm{n} / \mathrm{N}$} \\
\hline & & Derecha & Izquierda & $\chi^{2}$ & $p$ \\
\hline \multirow{17}{*}{ Entesis } & Deltoides & $3 / 6$ & $4 / 6$ & 0.34 & 0.55 \\
\hline & Dorsal ancho & $2 / 6$ & $2 / 6$ & $\mathrm{n} / \mathrm{c}$ & $\mathrm{n} / \mathrm{c}$ \\
\hline & Pectoral mayor & $3 / 6$ & $5 / 6$ & 1.5 & 0.22 \\
\hline & Redondo mayor & $1 / 5$ & $3 / 3$ & 1.06 & 0.3 \\
\hline & Braquiorradial & $4 / 6$ & $6 / 6$ & 2.4 & 0.12 \\
\hline & Braquial anterior & $4 / 6$ & $4 / 7$ & 0.1 & 0.72 \\
\hline & Bíceps braquial & $2 / 5$ & $3 / 7$ & 0.01 & 0.92 \\
\hline & Pronador cuadrado & $2 / 6$ & $3 / 6$ & 0.34 & 0.55 \\
\hline & Supinador & $4 / 6$ & $3 / 4$ & 0.07 & 0.77 \\
\hline & Glúteo mayor & $6 / 7$ & $6 / 7$ & $\mathrm{n} / \mathrm{c}$ & $\mathrm{n} / \mathrm{c}$ \\
\hline & Tuberosidad isquiática & $4 / 7$ & $4 / 7$ & $\mathrm{n} / \mathrm{c}$ & $\mathrm{n} / \mathrm{c}$ \\
\hline & Línea áspera & $2 / 7$ & $4 / 7$ & 1.16 & 0.28 \\
\hline & Gastrocnemios & $1 / 6$ & $3 / 6$ & 1.5 & 0.22 \\
\hline & Ligamento rotuliano & $1 / 5$ & $2 / 5$ & 0.47 & 0.49 \\
\hline & Poplíteo & $3 / 5$ & $2 / 6$ & 0.78 & 0.37 \\
\hline & Soleo & $3 / 5$ & $2 / 6$ & 0.1 & 0.74 \\
\hline & Aponeurosis plantar & $2 / 3$ & $1 / 6$ & 2.25 & 0.13 \\
\hline \multirow{6}{*}{$\begin{array}{l}\text { Complejo fun- } \\
\text { cional }\end{array}$} & Hombro & $9 / 23$ & $14 / 24$ & 1.7 & 0.18 \\
\hline & Codo & $10 / 17$ & $13 / 20$ & 0.22 & 0.63 \\
\hline & Muñeca-mano & $6 / 12$ & $6 / 10$ & 0.14 & 0.69 \\
\hline & Cadera & $10 / 14$ & $10 / 14$ & $\mathrm{n} / \mathrm{c}$ & $\mathrm{n} / \mathrm{c}$ \\
\hline & Rodilla & $10 / 30$ & $15 / 31$ & 1.42 & 0.23 \\
\hline & Tobillo-pie & $5 / 8$ & $4 / 12$ & 1.64 & 0.19 \\
\hline \multirow{2}{*}{ Miembros } & Superior & $25 / 52$ & $33 / 54$ & 1.81 & 0.17 \\
\hline & Inferior & $22 / 45$ & $19 / 43$ & 0.19 & 0.65 \\
\hline
\end{tabular}

$\mathrm{n} / \mathrm{N}=$ entesis con cambios/entesis observadas. No fue calculado el valor de $\chi 2$ en aquellos casos en donde el $\mathrm{n} / \mathrm{N}$ fue igual en cada lateralidad y tampoco en casos en donde el valor de toda una fila en la tabla de contingencia fue igual a 0.

nor (cadera), semimembranoso-semitendinoso, poplíteo y cuádriceps femoral (rodilla) y tríceps sural (tobillo-pie). La presencia de cambios por lateralidad no presenta diferencias significativas bajo ningún nivel de análisis anatómico (Tabla 5).

\section{DISCUSIÓN}

De acuerdo con los resultados obtenidos se observó que, para el cálculo de frecuencia de la presencia de cambios $(\mathrm{n} / \mathrm{N})$, en los dos métodos más de la mitad de las entesis analizadas fue afectada por al menos un cambio, pero mediante Co se registró un mayor porcentaje (Co: 69\% vs. HyM: 52\%). De modo inverso, el Índice de Afectación de Co fue menor (Co: 15\% vs. HyM: $23 \%$ ). El cambio entesial más frecuente en HyM fue la robusticidad y en Co la erosión en zona 2 , y en ambos métodos $c a .80 \%$ de la expresión de los CE fue mínima (i.e., HyM: leve; Co: 1). 
V. ROMANO Y A. SERNA/REV ARG ANTROP BIOL 22(1), 2020. doi:10.24215/18536387e012

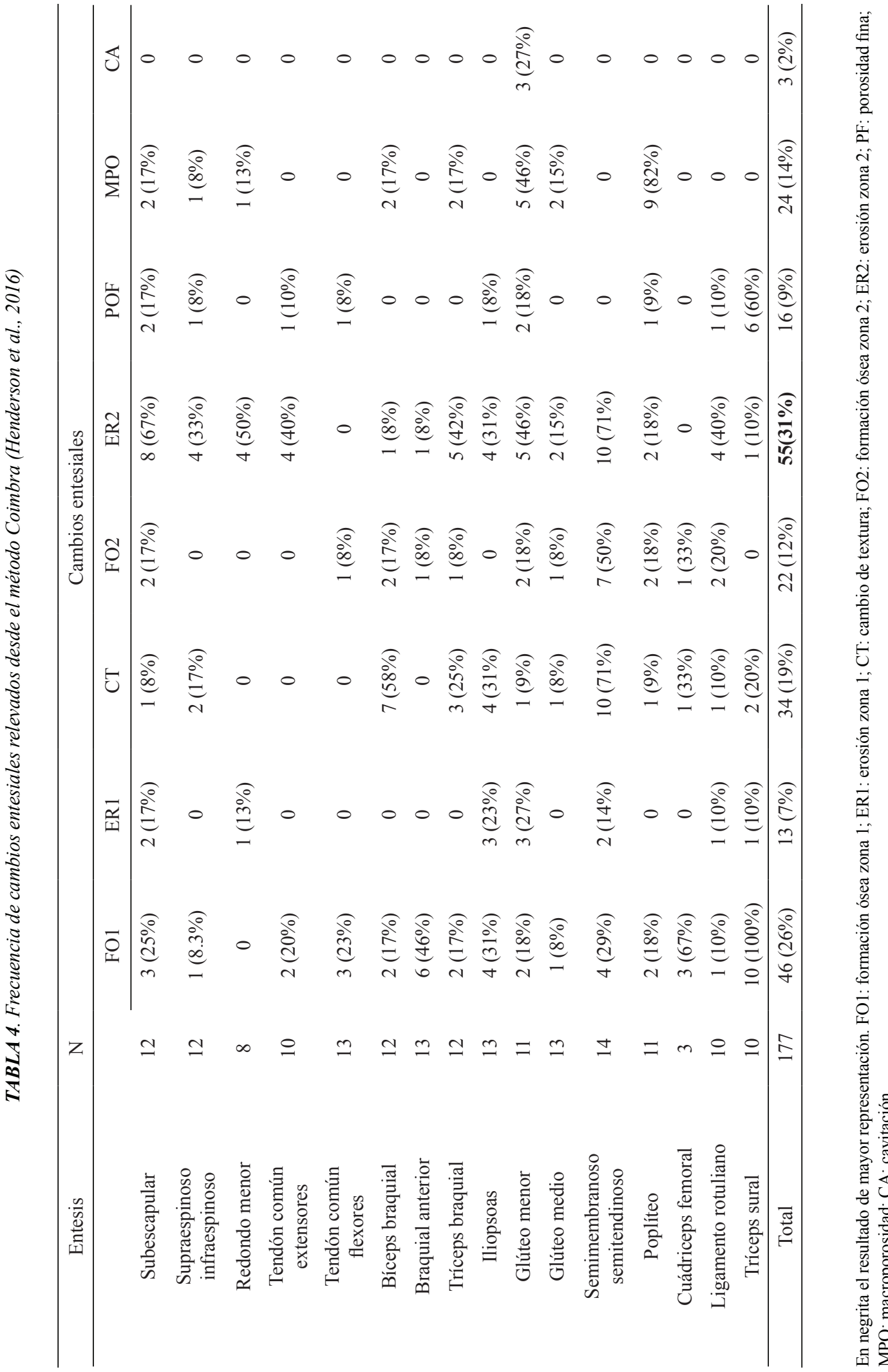




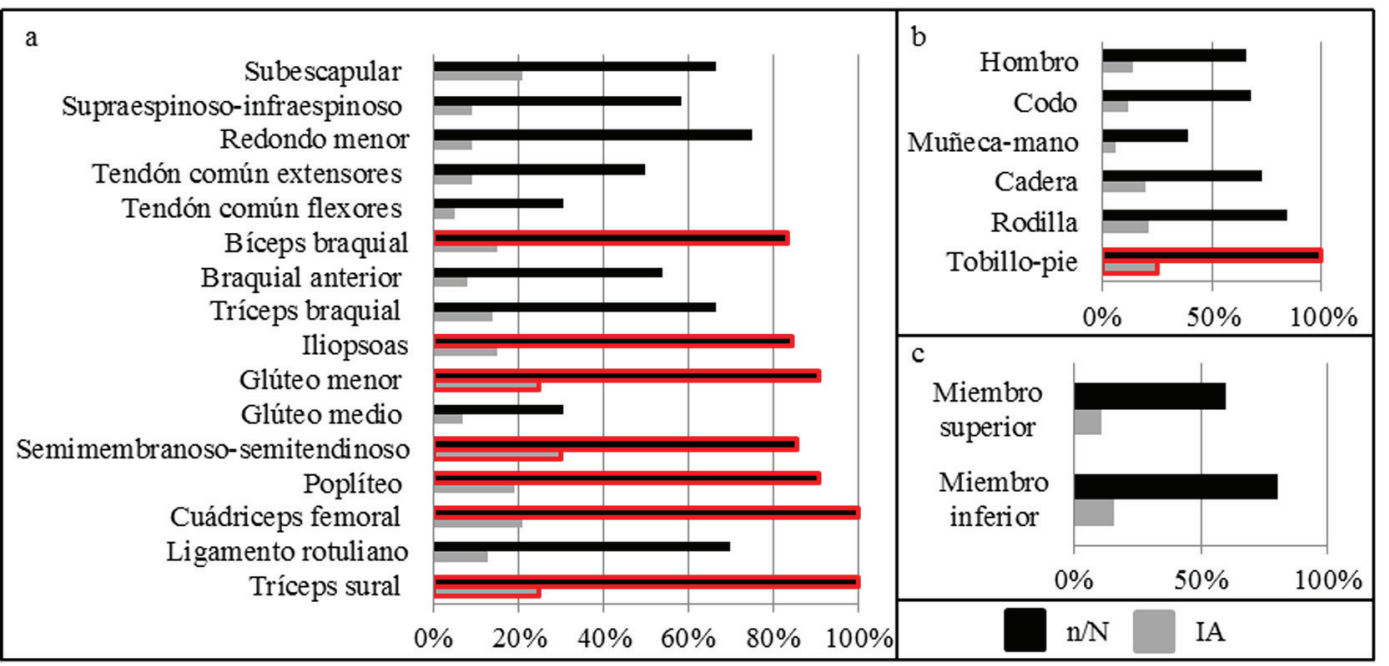

Fig. 4. Resultados desde el método Coimbra (Henderson et al., 2016) de presencia de cambios (n/N) e Índice de Afectación (IA) a distintos niveles: a) entesis; b) complejo funcionales; c) miembros. En color algunos porcentajes elevados que serán discutidos especialmente.

TABLA 5. Valores de $\chi 2$ para la presencia de cambios por lateralidad en el método Coimbra (Henderson et al., 2016)

\begin{tabular}{|c|c|c|c|c|c|}
\hline & & \multicolumn{2}{|c|}{$\mathbf{n} / \mathbf{N}$} & \multirow[b]{2}{*}{$\chi^{2}$} & \multirow[b]{2}{*}{ p } \\
\hline & & Derecha & Izquierda & & \\
\hline \multirow{16}{*}{ Entesis } & Subescapular & $2 / 5$ & $6 / 7$ & 2.74 & 0.09 \\
\hline & Supraespinoso-infraespinoso & $3 / 5$ & $4 / 7$ & 0.01 & 0.92 \\
\hline & Redondo menor & $3 / 4$ & $3 / 4$ & $\mathrm{n} / \mathrm{c}$ & $\mathrm{n} / \mathrm{c}$ \\
\hline & Tendón común extensores & $2 / 5$ & $3 / 5$ & 0.40 & 0.52 \\
\hline & Tendón común flexores & $2 / 6$ & $2 / 7$ & 0.03 & 0.85 \\
\hline & Bíceps braquial & $4 / 5$ & $6 / 7$ & 0.06 & 0.79 \\
\hline & Braquial anterior & $4 / 6$ & $3 / 7$ & 0.73 & 0.39 \\
\hline & Tríceps braquial & $5 / 6$ & $3 / 3$ & 1.50 & 0.22 \\
\hline & Iliopsoas & $5 / 6$ & $6 / 7$ & 0.01 & 0.9 \\
\hline & Glúteo menor & $4 / 5$ & $6 / 6$ & 1.32 & 0.25 \\
\hline & Glúteo medio & $2 / 6$ & $3 / 7$ & 0.12 & 0.72 \\
\hline & Semimembranoso-semitendinoso & $6 / 7$ & $6 / 7$ & $\mathrm{n} / \mathrm{c}$ & $\mathrm{n} / \mathrm{c}$ \\
\hline & Poplíteo & $5 / 6$ & $5 / 5$ & 0.91 & 0.33 \\
\hline & Cuádriceps femoral & $3 / 3$ & $0 / 0$ & $\mathrm{n} / \mathrm{c}$ & $\mathrm{n} / \mathrm{c}$ \\
\hline & Ligamento rotuliano & $4 / 5$ & $3 / 5$ & 0.47 & 0.49 \\
\hline & Tríceps sural & $4 / 4$ & $6 / 6$ & $\mathrm{n} / \mathrm{c}$ & $\mathrm{n} / \mathrm{c}$ \\
\hline \multirow{6}{*}{$\begin{array}{l}\text { Complejo } \\
\text { funcional }\end{array}$} & Hombro & $8 / 14$ & $13 / 18$ & 0.79 & 0.37 \\
\hline & Codo & $13 / 17$ & $12 / 20$ & 1.13 & 0.28 \\
\hline & Muñeca-mano & $4 / 11$ & $5 / 12$ & 0.06 & 0.79 \\
\hline & Cadera & $17 / 24$ & $21 / 27$ & 0.32 & 0.57 \\
\hline & Rodilla & $18 / 21$ & $14 / 17$ & 0.07 & 0.77 \\
\hline & Tobillo-pie & $4 / 4$ & $6 / 6$ & $\mathrm{n} / \mathrm{c}$ & $\mathrm{n} / \mathrm{c}$ \\
\hline \multirow{2}{*}{ Miembros } & Miembro superior & $25 / 42$ & $30 / 50$ & 0.00 & 0.92 \\
\hline & Miembro inferior & $33 / 42$ & $35 / 45$ & 0.01 & 0.96 \\
\hline
\end{tabular}

$\mathrm{n} / \mathrm{N}=$ entesis con cambios/entesis observadas. No fue calculado el valor de $\chi 2$ en aquellos casos en donde el $\mathrm{n} / \mathrm{N}$ fue igual en cada lateralidad y tampoco en casos en donde el valor de toda una fila en la tabla de contingencia fue igual a 0 . 
A pesar de la similitud de estos resultados generales, el análisis de los CE bajo distintos niveles anatómicos reveló diferencias importantes. De acuerdo con HyM, el complejo funcional más afectado es el de la cadera, seguido por los del miembro superior (codo, muñeca-mano y hombro, respectivamente), y a nivel de entesis la variación no sugiere ninguna tendencia anatómica (e.g., las entesis más afectadas pertenecen al miembro inferior -glúteo mayor- y al superior -braquiorradial-). En cambio, los resultados obtenidos con Co muestran una tendencia hacia una mayor afección en entesis y complejos asociados al miembro inferior. El complejo funcional tobillo-pie es el más comprometido, lo que contrasta con lo señalado por HyM ya que lo muestra como uno de los menos alterados.

A nivel metodológico, el análisis de datos se basó en la complementación de dos medidas de cuantificación aplicadas sobre distintos niveles anatómicos. La naturaleza acumulativa de los cambios en las superficies entesiales (Henderson y Nikita, 2016; Henderson et al., 2017; Villotte y Knüsel, 2013), plantea la importancia de considerar la cantidad y no solo la presencia de cambios. Por ejemplo, desde Co el $86 \%$ de las entesis del iliopsoas y semimembranoso-semitendinoso presentan al menos un cambio $(\mathrm{n} / \mathrm{N})$, no obstante, la cantidad de cambios registrada en cada grupo fue diferente (IA iliopsoas: 15\% vs. IA semiembranoso-semitendinoso: $30 \%$ ). Esto implica que las entesis del semimembranoso-semitendinoso se encuentran más afectadas que las del iliopsoas y, que caracterizarlas de la misma manera en base a una sola medida podría suponer una sobresimplificación del caso.

Si bien el énfasis de este trabajo está puesto en la comparación metodológica con fines descriptivos, así como en la aplicación de nuevas medidas de cuantificación, también resaltamos que la combinación de ambos métodos podría no ser aconsejable. En primer lugar, porque los métodos contemplan tipos de entesis con rasgos y ubicaciones anatómicas diferentes (i.e., entesis fibrosas y fibrocartilaginosas). En segundo lugar, los cambios que se registran en los elementos óseos y sus interpretaciones biomecánicas también difieren. En HyM las variables "robusticidad" y "lesión de estrés" se consideran como productos de microtraumas y la "osificación" de macrotraumas, y sus grados de expresión son directamente relacionados al impacto biomecánico recibido (Hawkey y Merbs, 1995), por lo que una alta frecuencia de robusticidad en grado leve implicaría la exposición reiterada a un estrés de baja intensidad biomecánica. En cambio, Co no establece un correlato preciso biomecánico per se para cada frecuencia de cambio y expresión en forma individual, sino que describe la variabilidad y distribución de los CE e infiere únicamente a partir de todo el conjunto de datos (Henderson et al., 2017; Michopoulou, Nikita y Henderson, 2017). En tercer lugar, el método HyM basa sus interpretaciones en la "Ley de remodelación ósea" (Wolff, 1986), que considera que el estrés al que es sometido el hueso genera un aumento del flujo sanguíneo que estimula a los osteoblastos, lo que resulta en una hipertrofia ósea y la consecuente modificación del área sometida al estrés (Hawkey y Merbs, 1995). En este sentido, Co se basa en conocimientos histológicos y clínicos actuales, que comprenden que las entesis fibrocartilaginosa en su zona interna son generalmente avasculares y, en su estado "sano/no modificado", presentan una morfología suave, circunscripta y sin forámenes venosos (Benjamin y McGonagle, 2001; Benjamin, Evans y Copp, 1986; Benjamin et al., 2002). Esto significa que el principio del aumento vascular en el que se basa HyM no sería aplicable en las entesis fibrocartilaginosas, principalmente por estar desprovistas de vasos sanguíneos, siendo los cambios producto de modificaciones del tendón y fibrocartílago cuya etiología puede deberse a diversos factores, entre ellos la actividad física (Benjamin y McGonagle, 2001; Villotte y Knüsel, 2013; Villotte et al., 2010).

\section{Consideraciones finales}

La comparación de dos metodologías de registro de cambios entesiales aquí realizada (HyM vs. Co) muestra contrastes en distintos niveles anatómicos, que pueden interpretarse como reflejo de las particularidades teóricas y analíticas de cada una de las metodologías. A nivel analítico, la complementación de dos medidas de cuantificación que refieren a distintos aspectos de los cambios entesiales ( $\mathrm{n} / \mathrm{N}$ : presencia de cambios e IA: "Índice de Afectación") permitió obtener una visión más completa sobre su expresión y variabilidad en los distintos ni- 
veles anatómicos. Por último, la aplicación de dos metodologías contrastantes permitió comprender mejor sus funcionamientos y planificar su consideración para futuros trabajos centrados en el estudio de los patrones de actividad.

\section{AGRADECIMIENTOS}

Queremos expresar nuestro agradecimiento a Luciano Prates por la lectura crítica del manuscrito. A los dos evaluadores anónimos quienes contribuyeron a mejorar substancialmente este trabajo con sus comentarios y sugerencias.

\section{LITERATURA CITADA}

Benjamin, M. y McGonagle, D. (2001). The anatomical basis for disease localization in seronegative spondyloarthropathy at entheses and related sites. The Journal of Anatomy, 199(5), 503-526. doi:10.1046/j.14697580.2001.19950503.x

Benjamin, M. y McGonagle, D. (2009). Entheses: tendon and ligament attachment sites. Scandinavian Journal of Medicine and Science in Sports, 19(4), 520-527. doi:10.1111/j.1600-0838.2009.00906.x

Benjamin, M., Evans, E. J. y Copp, L. (1986). The histology of tendon attachments to bone in man. Journal of Anatomy, 149, 89-100.

Benjamin, M., Kumai, T., Milz, S., Boszczyk, B. M., Boszczyk, A.A. y Ralphs, J. R. (2002). The skeletal attachment of tendons-tendon "entheses". Comparative Biochemistry and Physiology Part A: Molecular and Integrative Physiology, 133(4), 931-945. doi: 10.1016/ S1095-6433(02)00138-1

Bernal, V., González, P. N., Pérez, S. I. y Del Papa, M. C. (2004). Evaluación del error intraobservador en bioarqueología. Intersecciones en Antropología, 5, 129-140. doi:10.17139/raab.2016.0018.02.07

Buikstra, J. E. y Ubelaker, D. (1994). Standards for data collection from human skeletal remains. Faytteville, Estados Unidos. Arkansas Archaeological Survey Research Series, 44

Cohen, J. (1960). A coefficient of agreement for nominal scales. Educational Psychology Measurement, 20, 37 46. doi:10.1177/001316446002000104

Daniel, W. W. (1987). Bioestadística: base para el análisis de las ciencias de la salud. México: Limusa.

Hawkey, D. E. (1988). Use of upper extremity enthesopathies to indicate habitual activity patterns. Tesis de maestría inédita. Arizona State University. Tempe.

Hawkey, D. E. y Merbs, C. F. (1995). Activity-induced musculoskeletal stress markers (MSM) and subsistence strategy changes among ancient Hudson bay Eskimos. International Journal of Osteoarchaeology, 5(4), 324338. doi:10.1002/oa.1390050403

Henderson, C. Y. y Nikita, E. (2016). Accounting for multiple effects and the problem of small sample sizes in osteology: a case study focussing on entheseal changes. Archaeological and Anthropological Sciences, 8(4), 805-817. doi:10.1007/s12520-015-0256-1

Henderson, C. Y., Mariotti, V., Pany-Kucera, D., Villotte, S. y Wilczak, C. (2013). Recording specific entheseal changes of fibrocartilaginous entheses: initial tests using the Coimbra method. International Journal of Osteoarchaeology, 23(2), 152-162. doi:10.1002/oa.2287

Henderson, C. Y., Mariotti, V., Pany-Kucera, D., Villotte, S. y Wilczak, C. A. (2016). The new "Coimbra Method": a biologically appropriate method for recording specific features of fibrocartilaginous entheseal changes. International Journal of Osteoarchaeology, 26(5), 925-932. doi:10.1002/oa.2477

Henderson, C. Y., Mariotti, V., Santos, F., Villotte, S. y Wilczak, C. A. (2017). The new Coimbra method for recording entheseal changes and the effect of age-atdeath. BMSAP, 29(3-4), 140-149. doi:10.1007/s13219017-0185-x

Kapandji, A. (2006). Fisiología articular. Madrid, España: Médica Panamericana.

Landis, J. R. y Koch, G. G. (1977). The measurement of observer agreement for categorical data. Biometrics, 33, 159-174. doi: $10.2307 / 2529310$

Mariotti, V., Facchini, F. y Belcastro, M. G. (2007). The study of entheses: proposal of a standardized scoring method for twentythree entheses of the postcranial skeleton. Collegium Antropologicum, 1, 191-313.

Michopoulou, E., Nikita, E. y Henderson, C. Y. (2017). A test of the effectiveness of the Coimbra method in capturing activity-induced entheseal changes. International Journal of Osteoarchaeology, 27(3), 409-417. doi:10.1002/oa.2564

Prates, L., Serna, A., Mange, E., López, L., Romano, V., Di Lorenzo, M., Saghessi, D. y González Venanzi, L. (2019). Ocupaciones residenciales y entierros humanos en Negro Muerto 3 (valle del río Negro, Norpatagonia). Magallania, 47(1), 159-176.

Robb, J. E. (1998). The interpretation of skeletal muscle sites: a statistical approach. International Journal of $\mathrm{Os}_{\mathrm{s}}$ teoarchaeology, 8, 363-377.

Scheuer, L. y Black, S. (2000). Developmental juvenile osteology. Londres, Reino Unido: Elseiver Academic Press.

Serna, A. y Romano, V. (2018). Rescates bioarqueológicos en el valle medio del río Negro (Provincia de Río Negro): el potencial informativo del registro altamente perturbado. Revista Argentina de Antropología Biológica, 20(2). doi:10.17139/raab.2018.0020.02.03

Stirland, A. J. (1998). Musculoskeletal evidence for activity: problems of evaluation. International Journal of Osteoarchaeology, 8(5), 354-362. doi:10.1002/ (S ICI) $1099-1212(1998090) 8: 5<354$ : A ID $\mathrm{OA} 432>3.0 . \mathrm{CO} ; 2-3$

Testut, L. y Latarjet, A. (1954). Compendio de anatomía descriptiva. Barcelona, España: Salvat.

Villotte, S. y Knüsel, C. J. (2013). Understanding entheseal changes: definition and life course changes. International Journal of Osteoarchaeology, 23(2), 135-146. doi:10.1002/oa.2289

Villotte, S., Assis, S., Cardoso, F. A., Henderson, C. Y., Mariotti, V., Milella, M. y Jurmain, R. (2016). In search of consensus: terminology for entheseal changes (EC). International Journal of Paleopathology, 13, 49-55. doi:10.1016/j.ijpp.2016.01.003

Villotte, S., Castex, D., Couallier, V., Dutour, O., Knüsel, C. J. y Henry-Gambier, D. (2010). Enthesopathies as occupational stress markers: evidence from the upper limb. American Journal of Physical Anthropology, 142(2), 224-234. doi:10.1002/ajpa.21217

White, T. y Folkens, P. A. (2005). The human bones manual. Londres, Reino Unido: Elsevier Academic Press.

Wolff, J. (1986). The law of bone remodeling. Berlin, Heidelberg: Springer-Verlag. 